\title{
Bagging Ensemble Model for Prediction of Dead Oil Viscosity
}

\author{
Bharat B. Gulyani, B. G. Prakash Kumar, and Arshia Fathima
}

\begin{abstract}
Dead oil viscosity is a critical design factor for oilfields and refineries. From available literature, crude oil viscosity is found to be a strong function of pressure, temperature, bubble-point pressure, gas-oil ratio, gas gravity, and oil gravity. Oil viscosity is generally determined from laboratory experiments and empirically derived correlations.

Reliable measurements of dead oil viscosity are difficult to obtain due to lack of lab equipment or liquid samples. Based on API oil gravity \& temperature, various correlations have been used to predict dead oil viscosity. In addition to correlations, recently data mining techniques like Artificial Neural Networks (ANN) and Support Vector Machines (SVM) have been used to predict petroleum viscosity. The aim of this paper is to introduce the ensemble model of bagging as an important data mining technique to predict dead oil viscosity. The ensemble model predicted the viscosity accurately with a correlation coefficient of 0.99 , an accuracy that is comparable to that of ANN as found in literature. It was also observed that bagging lowered the relative error of the base classifier (ANN) from $10 \%$ to about $8 \%$, thereby stabilizing the ANN while retaining its accuracy.
\end{abstract}

Index Terms-API gravity, bagging, data mining, dead oil viscosity.

\section{INTRODUCTION}

Crude oil viscosity is an important physical property that influences various operations in oil fields and refineries. The crude oil viscosity depends on its origin, type and chemical composition. Oil viscosity is measured in PVT laboratories only at its reservoir temperature. Dead oil viscosity is measured at atmospheric pressure (no gas in solution) and system temperature, which makes it difficult to obtain reliable measurements due to lack of lab equipment or liquid samples [1]. However, due to viscosity variability under different operating conditions, there is a need for measuring techniques or correlations to obtain the viscosity value as a function of important parameters. The correlations so far developed have been proposed for specific range of operating conditions and are limited in application to region corresponding to the origin of the crude oil samples used for the derivation of the correlation.

The prediction of crude oil viscosity (dead oil, saturated \& under-saturated oil viscosities) has shown to improve through use of data mining techniques including Artificial Neural Networks (ANN) and Support Vector Machines (SVM) as

Manuscript received October 26, 2016; revised January 20, 2017.

Bharat B. Gulyani and B. G. Prakash Kumar are with Department of Chemical Engineering, BITS Pilani, Dubai Campus, Academic City, Dubai 345055, UAE (e-mail: gulyanibb@gmail.com, prakash@dubai.bits-pilani.ac.in).

Arshia Fathima is with Nanolabs, Alfaisal University (e-mail: arshiafathima92@gmail.com). observed from literature. This paper aims to introduce ensemble method of bagging to predict dead oil viscosity which is shown to have an accuracy comparable to that of ANN used in literature. Ensemble methods work well with smaller data sets and avoid over-fitting as they average out the results. These models solve problems by having multiple base classifies that have their local optima and then average the predictions thereby reducing risk of choosing wrong classifier and reduce variations in predictability due to minor changes in data sets. Hence ensembles can boost performance of unstable classifiers like ANN [2].

\section{LITERATURE REVIEW}

Recent studies conducted on use of neural networks for prediction of dead oil viscosity have shown to perform better than the existing correlations. A study on Nigerian crude oils developed a neural network based on the PVT data of the samples obtained to predict the sample viscosity. The neural networks developed had a correlation coefficient of 0.99 and performed better than existing empirical correlations [3], [4]. Similar studies with neural networks to predict viscosity of Iranian oils [5]-[7] and Omani oils [8] have shown to perform better than existing correlations. Besides this the entire viscosity curves have also been shown to be accurately predicted for Canadian oilfields using neural networks and SVM [9] thereby establishing the reliable use of data mining techniques to predict crude oil viscosity.

TABLE I: DESCRIPTIVE STATISTICS OF INPUT AND OUTPUT DATA USED

\begin{tabular}{llll}
\hline \hline Statistics & API & Temperature & Viscosity \\
\hline Mean & 26.5 & 160 & 8.56 \\
Sample Variance & 9.2 & 1405.15 & 49.45 \\
Range & 10 & 120 & 41.01 \\
\hline \hline
\end{tabular}

\section{Methodology}

To build a robust data prediction model for dead oil viscosity, the data set was developed by using the correlations found in literature as given in Section III. The correlations from literature were used to calculate the dead oil viscosity for the specific range of API from 21.5 - 31.5 (in steps of 0.5) and temperature range from $100-220 \mathrm{~F}$ (increment unit of $10 \mathrm{~F}$ ). The arithmetic mean of the viscosity calculated from the correlations was then used an input to the data mining model along with temperature and API gravity. Statistics for the data developed is given in Table I. The reservoir pressures were not taken into consideration as the dead oil viscosity is measured at atmospheric pressures. The bagging model was 
developed using the open source software called WEKA (Waikato Environment for Knowledge Analysis) [10].

\section{A. Correlations used for Dataset Development}

The correlations used to calculate the dead oil viscosity for given temperature and API gravity to form the data set are described below:

1) Beal's Correlation [11]: This correlation, developed from crude oil data of California, expresses viscosity as a function of API gravity and temperature. The correlation is applicable for API gravity range of $10-52$ and temperature range 100-220 $\mathrm{F}$ [12].

$$
\mu_{o d}=\left(0.32+\frac{1.8 \times 10^{7}}{A P I^{4.53}}\right)\left(\frac{360}{T+200}\right)^{a}
$$

where

$$
a=\operatorname{anti} \log \left(0.43+\frac{8.33}{A P I}\right)
$$

2) Beggs-Robinson Correlation [13]: Formulated in 1975 for API range of 16-58 and temperature range 70-295F [12], this correlation is based crude oils of unknown location.

$$
\mu_{\text {od }}=10^{x}-1
$$

where

$$
x=10^{(3.0324-0.02023 A P I)} \times T^{-1.163}
$$

3) Glaso Correlation [14]: This correlation is developed for North Sea oils for specified ranges of 20-48 API and temperatures of 50-300 F [12].

$$
\begin{aligned}
\mu_{o d}= & \left(3.141 \times 10^{10}\right) \times T^{-3.4444} \\
& \times \log (A P I)^{[10.313 \log (T)-36.447]}
\end{aligned}
$$

4) Kartoatmodjo et al. Correlation: Following correlation approximates dead oil viscosity for API ranges 14.4 -59 and temperature range $80-320 \mathrm{~F}$ [12].

$$
\begin{aligned}
\mu_{\text {od }}= & 16 \times 10^{8} \times T^{-2.8177} \\
& \times\left[\log (A P I)^{(5.7526 \log (T)-26.9718)}\right]
\end{aligned}
$$

Above equation is modified for viscosity estimation for medium oils:

$$
\begin{aligned}
\mu_{o d}= & 220.15 \times 10^{9} \times T^{-3.5660} \\
& \times\left[\log (A P I)^{(12.5428 \log (T)-45.7874)}\right]
\end{aligned}
$$

e) Naseri et al. Correlation: This correlation is developed in Iran for dead oil viscosity estimates in API range 17-44 and temperature range 105-295F [12].

$$
\mu_{\text {od }}=\operatorname{anti} \log \left(\begin{array}{l}
11.2699 \\
-4.298 \log (A P I) \\
-2.052 \log (T)
\end{array}\right)
$$

\section{B. Bagging Model for Data Mining}

An ensemble model trains multiple base classifiers and averages their results for prediction or classification (depending upon the application) to give the final output. Bootstrap Aggregating or bagging is an ensemble method that manipulates instances by using bootstrap sampling for getting the training and testing sets from feed data. Bootstrap sampling involves sampling ' $n$ ' instances ' $n$ ' times with replacement. In this way, all the data will be used for training and validating the data giving a generalized model thereby avoiding the issues of errors and overfitting [15].

\section{Performance Measures}

The performance measures used in this study were Root Mean Squared Error (RMSE), correlation coefficient, Mean Absolute Error (MAE) and Relative Absolute Error (RAE). The smaller the errors with higher correlation coefficient, the better the performance of the model. Scatter and line plots were also used for visual comparison between the correlations and the data mining models for their predictive performance.

The RMSE is calculated by the following formula [16]:

$$
\mathrm{RMSE}=\sqrt{\sum_{i=1}^{N} \frac{\left(p_{i}-a_{i}\right)}{N}}
$$

where $p_{i}$ is the predicted value for the ith instance, $\mathrm{a}_{\mathrm{i}}$ is the actual value for the ith instance and $\mathrm{N}$ is the total number of instances in the given data set.

The correlation coefficient measures the degree of linear relation between 2 variables. A correlation coefficient of 0 implies no correlation between variables while a value of 1 implies perfect correlation. The correlation coefficient between actual and predicted variables [16] is given by:

$$
R^{2}=S_{p a} /\left(\sqrt{S_{p} S_{a}}\right)
$$

where $\bar{a}$ and $\bar{p}$ are the averages respectively, and

$$
\begin{gathered}
S_{p a}=\sum_{i=1}^{N}\left(p_{i}-\bar{p}\right)\left(a_{i}-\bar{a}\right)(N-1) \\
S_{p}=\frac{\sum_{i=1}^{N}\left(p_{i}-\bar{p}\right)^{2}}{N-1} \\
S_{a}=\frac{\sum_{i=1}^{N}\left(a_{i}-\bar{a}\right)^{2}}{N-1}
\end{gathered}
$$

Mean Absolute Error (MAE) is the absolute average between the predicted and actual values and it reduces the bias towards large events unlike RMSE. The equation for MAE [17]:

$$
\mathrm{MAE}=\frac{1}{N} \sum_{i=1}^{N}\left|a_{i}-p_{i}\right|
$$

where $p_{i}$ is the predicted value for the $i^{\text {th }}$ instance, $a_{i}$ is the actual value for the $i^{\text {th }}$ instance and $N$ is the total number of instances in the given data set. 
Relative Absolute Error (RAE) is the relative equivalent of MAE [17] and is given by:

$$
\mathrm{RAE}=\frac{1}{\mathrm{~N}} \sum_{i=1}^{N} \frac{\left|a_{i}-p_{i}\right|}{a_{i}}
$$

where $p_{i}$ is the predicted value for the $i^{\text {th }}$ instance, $a_{i}$ is the actual value for the $i^{\text {th }}$ instance and $\mathrm{N}$ is the total number of instances in the given data set.

\section{RESULTS}

Based on default settings of Bagging \& Multilayer Perceptron (ANN) models in WEKA as given in Table II, the proposed model was built with 10 -fold cross validation. The ANN in WEKA uses sigmoid function with backpropagation algorithm for classification/prediction. As seen from the performance measures given in Table III, the ensemble model (bagging with ANN) improves the performance of the ANN in prediction of dead oil viscosity.

It was also observed that the bagging ensemble model has a more stable predictability unlike the ANN model for dead oil viscosity for temperatures over $150 \mathrm{~F}$ as shown in Fig. 1 and Fig. 2(a) - 2(c) for temperatures $190-210$ F. This can be attributed to the large variability in the data set inducing instability in the ANN model. From these, we can safely conclude that ensemble models would provide for stable performance in future applications in oilfields and refineries.

TABLE II: MODEL PARAMETERS FOR PREDICTION OF DEAD OIL VISCOSITY

\begin{tabular}{ll}
\hline \hline Model & Parameter Settings \\
\hline & $\begin{array}{l}\text { Hidden Layers }=\mathrm{a}=1 \text { (This is a } \\
\text { wildcard value equal to } \\
\text { (attributes+class) } / 2\end{array}$ \\
Lultilayer Perceptron (ANN) & $\begin{array}{l}\text { Nearning Rate }=0.3 ; \text { Momentum }=0.2 \\
\text { Normalize Numeric Class = True } \\
\text { Training Time }=500 ; \\
\text { Validation Threshold }=20\end{array}$ \\
& Bag Size Percent $=100$ (This is the \\
& size of each bag, as a percentage of the \\
& training set size) \\
Base Classifier $=$ Multilayer \\
-Bagging with ANN as base \\
classifier
\end{tabular}

\section{CONCLUSIONS}

Dead oil viscosity is a crucial design factor in the oil industry and is an area of research interest for development of accurate prediction models to improve the design process. Correlations have been developed based on region specific data sets that may not be applicable to all the oil producing regions due to variation in nature of the oils. Recently, data mining techniques have been applied to predict the dead oil viscosity with improved performance as compared to the correlations. In this study, a generalized ensemble bagging model based on data from the correlations to predict dead oil viscosity was explored. The ensemble model had an equivalent prediction capability as that of ANN but exhibited superior performance with regards to stability as reflected in RMSE when compared with ANN. Future studies on ensemble techniques should be conducted on datasets containing consolidated data from various regions so as to develop a robust technique to predict the dead oil viscosity.

TABLE III: MODEL PERFORMANCE COMPARISON FOR PREDICTION OF DEAD OIL VISCOSITY

\begin{tabular}{lllll}
\hline \hline Model & $\begin{array}{l}\text { Correlation } \\
\text { Coefficent }\end{array}$ & RMSE & MAE & RAE(\%) \\
\hline $\begin{array}{l}\text { Multilayer } \\
\text { Perceptron (ANN) }\end{array}$ & 0.99 & 0.77 & 0.55 & 10.9 \\
$\begin{array}{l}\text { Proposed } \\
\text { Ensenmble Model }\end{array}$ & 0.99 & 0.62 & 0.44 & 8.74 \\
\hline \hline
\end{tabular}

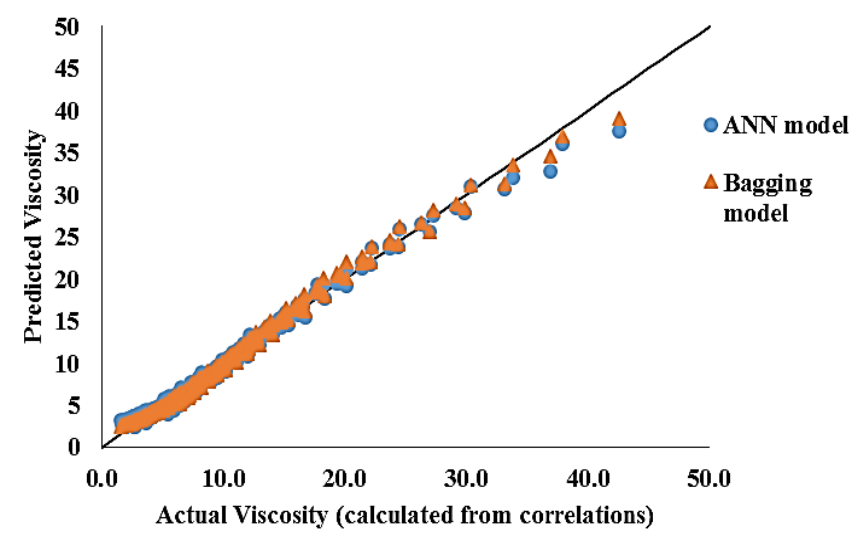

Fig. 1. General performance comparison for prediction of viscosity by ANN and Bagging models.

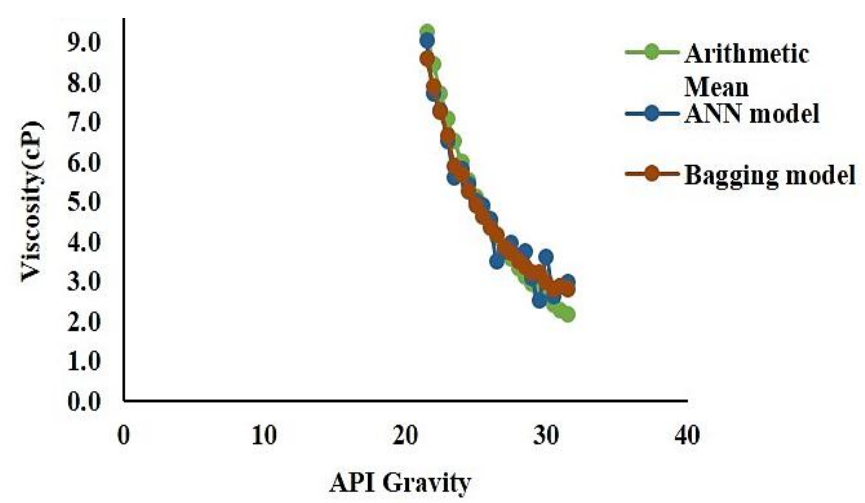

Fig. 2(a). Performance comparison for prediction of viscosity by the models at $\mathrm{T}=190 \mathrm{~F}$.

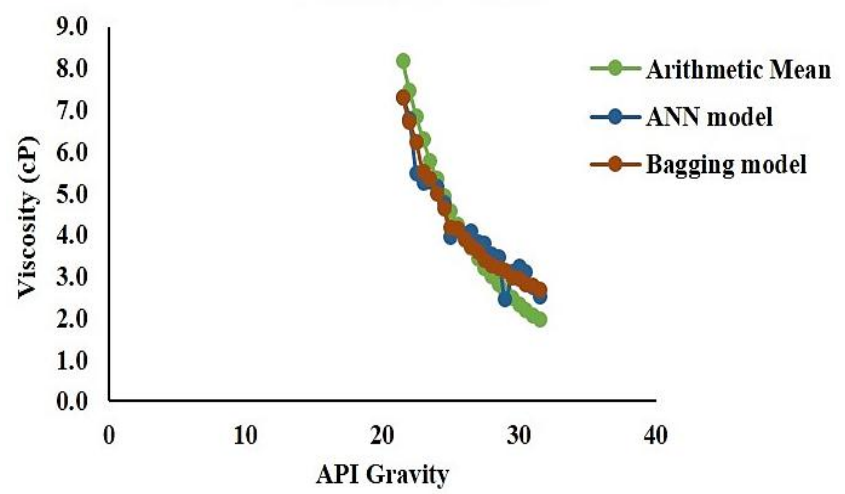

Fig. 2(b). Performance comparison for prediction of viscosity by the models at $\mathrm{T}=200 \mathrm{~F}$. 


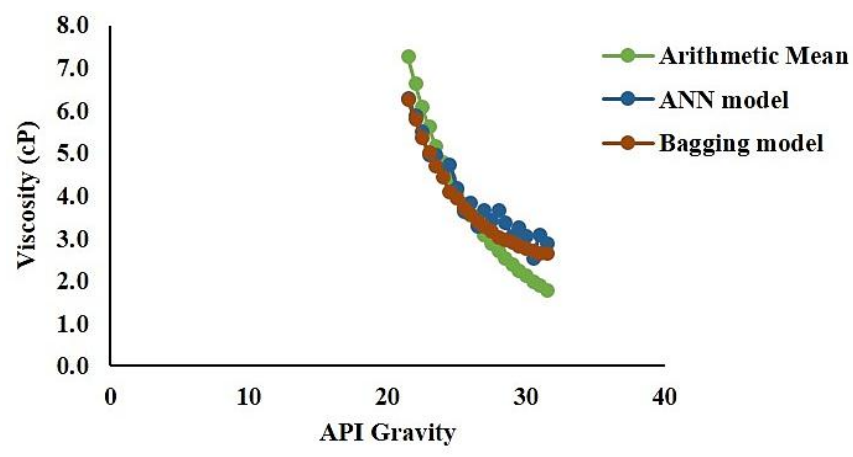

Fig. 2(c). Performance comparison for prediction of viscosity by the models at $\mathrm{T}=210 \mathrm{~F}$.

\section{REFERENCES}

[1] E. A. Edreder and K. M. Rahuma, "Testing the performance of some dead oil viscosity correlations," Petroleum \& Coal, vol. 54, no. 4, pp. 397-402, 2012.

[2] Z. Zheng and B. Padmanabhan, "Constructing Ensembles from data envelopment analysis," INFORMS Journal on Computing, vol. 19, no. 4, pp. 486-496, 2007.

[3] D. A. Deng, O. A. Falode, and O. Omole, "Prediction of Nigerian crude oil viscosity using Artificial Neural Network," Petroleum and Coal, vol. 51, no. 3, pp. 181-188, Jul. 2009.

[4] F. A. Makinde, C. T. Ako, O. D. Orodu, and I. U. Asuquo, "Prediction of crude oil viscosity using feed-forward back-propagation neural network (FFBPNN)," Petroleum \& Coal, vol. 54, no. 2, pp. 120-131, 2012.

[5] A. Hemmati-Sarapardeh, S.-M.-J. Majidi, B. Mahmoudi, A. Ahmad Ramzani S., and A. H. Mohammadi, "Experimental measurement and modeling of saturated reservoir oil viscosity," Korean J. Chem. Eng., vol. 31, no. 7, pp. 1253-1264, May 2014.

[6] M. S. Lashkenari, M. Taghizadeh, and B. Mehdizadeh, "Viscosity prediction in selected Iranian light oil reservoirs: Artificial neural network versus empirical correlations," Pet. Sci., vol. 10, no. 1, pp. 126-133, Feb. 2013.

[7] A. Naseri, S. Yousefi, A. Sanaei, and A. Gharesheikhlou, "A neural network model and an updated correlation for estimation of dead crude oil viscosity," Brazilian Journal of Petroleum and Gas, vol. 6, no. 1, 2012.

[8] T. K. A. Wahaibi, F. S. Mjalli, and A. A. A. Hashmi, "Viscosity correlations for light Omani crude using artificial neural networks,' International Journal of Petroleum Engineering, vol. 1, no. 1, p. 92, 2014.

[9] M. A. Al-Marhoun, S. Nizamuddin, A. A. A. Raheem, S. S. Ali, and A. A. Muhammadain, "Prediction of crude oil viscosity curve using artificial intelligence techniques," Journal of Petroleum Science and Engineering, vol. 86-87, pp. 111-117, May 2012.

[10] M. Hall, E. Frank, G. Holmes, B. Pfahringer, P. Reutemann, and I. H. Witten, "The WEKA data mining software: An update," SIGKDD Explorer Newsletters, vol. 11, no. 1, pp. 10-18, Nov. 2009.

[11] C. Beal, "The viscosity of air, water, natural gas, crude oil and its associated gases at oil field temperatures and pressures," Transactions of the AIME, vol. 165, no. 1, pp. 94-115, Dec. 1946.
[12] H. M. Manzar Sattarina, H. Modarresi, M. Bayat, and M. Teymori, "New viscosity correlations for dead crude oils," Petroleum and Coal, vol. 49, no. 2, pp. 33-39, 2007.

[13] H. D. Beggs and J. R. Robinson, "Estimating the viscosity of crude oil systems," Journal of Petroleum Technology, vol. 27, no. 9, pp. 1140-1141, Sep. 1975.

[14] O. Glaso, "Generalized pressure-volume-temperature correlations," Journal of Petroleum Technology, vol. 32, no. 5, pp. 785-795, May 1980.

[15] P.-N. Tan, M. Steinbach, and V. Kumar, Introduction to Data Mining, 1st ed., Boston, MA, USA: Addison-Wesley Longman Publishing Co., Inc., 2005.

[16] I. H. Witten and E. Frank, Data Mining: Practical Machine Learning Tools and Techniques with Java Implementations, San Francisco, CA, USA: Morgan Kaufmann Publishers Inc., 2000.

[17] N. D. Bennett et al., "Characterizing performance of environmental models," Environmental Modelling \& Software, vol. 40, pp. 1-20, Feb. 2013.

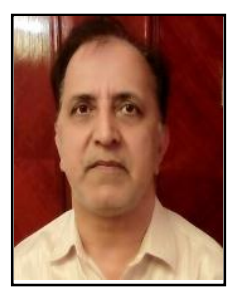

Bharat B. Gulyani had his bachelor, masters and doctoral degrees from University of Roorkee, India (now IIT Roorkee) in the field of chemical engineering. He has more than 20 years of research experience ad had published and presented at various conferences more than 30 research papers. He is currently Associate professor at Birla Institute of Technology and Science at their Dubai Campus.

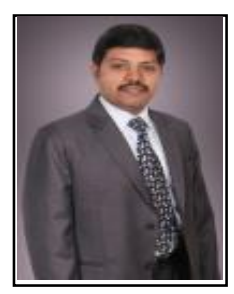

B. G. Prakash Kumar had his bachelor from Siddagana Institute of Technology, his master from Annamalai University and his doctoral degree from Anna University, India in the field of chemical engineering.

He has industrial experience as a process engineer with Grasim Industries, India and academic experience as a lecturer. Currently, he is Assistant Professor at Birla Institute of Technology and Science at their Dubai Campus. Actively involved in research, his interests include activated carbons synthesis, solar photo catalysis, wastewater treatment and separation processes.

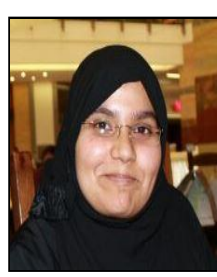

Arshia Fathima has received her bachelors in chemical engineering and computer science from BPDC, UAE in 2014 and her masters in chemical engineering with specialization in product development from UC Berkeley, USA in 2015.

Currently, she is pursuing research at the Nanolabs, Alfaisal University under Dr. Edreese Alsharaeh. Actively involved in research, she has published 5 papers until date in conferences and journals. Her research interests include desalination, water treatment, brine and other wastewater management. 